\title{
¿Son los Gobiernos locales más eficientes cuando su coalición política está en el Gobierno central? Un estudio para el caso de las municipalidades en Chile* \\ Are local governments more efficient as the same political coalition governs? A study among Chilean municipalities
}

\author{
José MARTÍNeZ** \\ CÉSAR SALAZAR*** \\ LuIS AMÉSTICA-RIVAS****
}

\begin{abstract}
Resumen
Últimamente, se ha generalizado una desconfianza en relación con la gestión de los recursos públicos. Este trabajo mide la eficiencia técnica de las municipalidades en Chile, haciendo especial énfasis en aquellos factores de tipo político que inciden en la eficiencia en la gestión local. En particular, el foco es puesto en la relación entre la ideología política del Gobierno local con el Gobierno central. Para ello, se utilizan datos de panel de municipalidades chilenas para los años 2010-2016. Las estimaciones siguen dos etapas. Primero, se mide la eficiencia mediante el Análisis Envolvente de Datos. Segundo, se estima un modelo Probit Fraccional para explicar estos niveles. Las estimaciones señalan que en promedio las municipalidades proveen un $49 \%$ menos de bienes y servicios que el óptimo. Niveles de eficiencia más altos se obtienen en comunas más grandes, urbanas y con mayor participación del Fondo Común Municipal. La eficiencia aumenta cuando el alcalde se presenta a reelección y se reduce en períodos en
\end{abstract}

* Los autores agradecen los valiosos aportes realizados por dos árbitros anónimos y el editor. Errores son de exclusiva responsabilidad de los autores.

** Programa de Magíster en Gestión de Empresas, Facultad de Ciencias Empresariales, Universidad del Bío-Bío. Chillán, Chile. Email: jhmartin@egresados.ubiobio.cl

*** Departamento de Gestión Empresarial, Facultad de Ciencias Empresariales, Grupo de Investigación Análisis Económico Sectorial Aplicado, Grupo de Investigación en Agronegocios, y Centro de Estudios Ñuble, Universidad del Bío-Bío, Chillan, Chile, Núcleo de Investigación en Economía Ambiental y de los Recursos Naturales (NERE) EfD center Chile. E-mail: csalazar@ubiobio.cl

**** Departamento de Gestión Empresarial, Facultad de Ciencias Empresariales, y Centro de Estudios Ñuble Universidad del Bío-Bío. Chillan, Chile, Grupo de investigación en Dirección universitaria de la Universidad Politécnica de Cataluña, España. Email: lamestica@ubiobio.cl

Received: November, 2018. Accepted: January, 2020. 
que gobierna una coalición de izquierda. Esto último se ve atenuado por un mayor grado de influencia del consejo municipal, sugiriendo una incidencia de la relación entre el Gobierno central y local en la eficiencia.

Palabras clave: Eficiencia, municipalidades, DEA, gestión, política.

Clasificación JEL: D22, H72, C14.

\begin{abstract}
Lately, questionings have emerged on how public resources are managed. This work calculates the technical efficiency of municipalities in Chile, with special emphasis on the political factors that drive efficiency in the management of local resources. In particular, focus is placed on the relationship between political ideology of the local government and central government. To do this, we use a panel data of Chilean municipalities for the years 2010-2016. The methodology follows two stages. First, we estimate efficiency scores by the Data Envelopment Analysis (DEA) approach. Second, a Fractional Probit model is estimated to explain these scores. The main results show that, on average, municipalities produce $49 \%$ achieve $49 \%$ less of output than the optimal. Higher levels of efficiency are obtained in larger, urban communes and with greater participation in the Municipal Common Fund. Efficiency increases when the mayor presents himselffor a re-election and is reduced in the periods in which a left coalition governs. The later effect is reduced to the extent that the majority of the municipal council share the same ideology of the national government, suggesting an effect of the relationship between the central and local government on efficiency levels.
\end{abstract}

Key words: Efficiency, municipalities, DEA, management, politics.

JEL Classification: D22, $H 72, C 14$.

\title{
1. INTRODUCCIÓN
}

Eficiencia se define como la capacidad de producir una determinada cantidad de producto con una cantidad mínima de insumos, gasto o esfuerzo (Sickles and Zelenyuk, 2019). Durante el último tiempo se han ido generalizando cada vez más en la población sentimientos como la intranquilidad y la desconfianza con relación a que no siempre los recursos públicos son empleados de manera efectiva y eficiente. Los Gobiernos locales, representados por las municipalidades, tienen por finalidad satisfacer las necesidades de la comunidad local y asegurar su participación en el progreso económico, social y cultural de sus respectivas comunas. Dado el incremento progresivo en los niveles de recursos generados o transferidos a los Gobiernos locales en los últimos años, cabe analizar si estos 
recursos están siendo utilizados de manera eficiente por quienes están a cargo de su administración. Una de las formas tradicionales utilizadas para dar a conocer a la ciudadanía como ha sido la gestión de las municipalidades ha sido mediante la cuenta pública. En esta actividad se informa a la comunidad en qué han sido utilizados los fondos municipales. Sin embargo, no es posible emitir un juicio si los montos desembolsados se relacionan con una buena o mala gestión por parte de los administradores locales.

De esta manera y en torno al principio de accountability, en una noción amplia de su significado (Morales-Trapp, 2016; Raupp \& de Pinho, 2013), es indispensable generar información a la ciudadanía en relación con la eficiencia de la gestión municipal de su comuna. Este artículo tiene como objetivo evaluar el desempeño de la gestión de los Gobiernos locales en Chile, a través de un análisis de la eficiencia técnica y determinar los factores que influencia este resultado, haciendo especial énfasis en aquellos de tipo político. En particular, el foco es puesto en la relación entre la ideología política del Gobierno local con el Gobierno central. Para aquello, se estiman los niveles de eficiencia para cada municipalidad utilizando el Análisis Envolvente de datos (DEA) (Charnes, Cooper, \& Rhodes, 1978) teniendo como principales fuentes de información el Servicio Nacional de Información Municipal (SINIM) y el Servicio Electoral (SERVEL) de Chile. Para medir los efectos de distintos factores sobre los niveles de eficiencia municipal incluyendo factores de orden político, se estima un modelo Probit Fraccional para datos de panel (Papke \& Wooldridge, 2008). Este modelo tiene la particularidad de ajustarse a variables dependientes acotadas entre cero y uno, como es el caso de la medición de la eficiencia la cual se calcula como un porcentaje relativo a la municipalidad más eficiente. Para control por potenciales factores no observados que podrían estar correlacionados con los niveles de eficiencia y factores afectando este indicador, se sigue la estrategia propuesta por Chamberlin (1980) quien asume una distribución de la heterogeneidad individual condicionada a los promedios de las variables explicativas. Alternativamente, se estima un modelo log od ratios el cual permite controlar directamente por efectos fijos a nivel de municipios.

La literatura abordando la medición de los niveles de eficiencia en la gestión de los Gobiernos locales y la influencia de factores de tipo económico, social y de gestión en este desempeño está bien documentada (ver por ejemplo Kalseth \& Ratts\&, 1995; Borger \& Kerstens, 1996; Worthington, 2000; Sampaio de Sousa, Cribari-Neto, \& Stosic, 2005; Loikkanen \& Susiluoto, 2005; Afonso \& Fernandes, 2006; Balaguer-coll, Prior, \& Tortosa-ausina, 2007; Nijkamp \& Suzuki, 2009; Benito et al., 2010; Bosch \& Espasa, 2012; Balaguer-coll, Prior, \& Tortosa-ausina, 2013; Da Cruz \& Marquez, 2014; El Mehdi, R. \& Hafner, C.M., 2014). Sin embargo, la literatura explorando factores políticos en la eficiencia en la gestión municipal es relativamente más reciente. Como también, algunos estudios que han buscado la relación entre eficiencia de las autoridades locales y el nivel de corrupción (Bostan et al., 2018). La mayoría de los estudios en este ámbito se enmarcan en examinar el efecto de la competencia electoral en la eficiencia en la gestión municipal (Calvalcante, 2013; Pacheco et al., 2014; Yusfany, 2015; Soto, 2016). 
La literatura es más escasa en lo que respecta a la relación entre la posición ideológica del alcalde y su apoyo político en los niveles de eficiencia. Una excepción es Št’astná \& Gregor (2015), quienes estimaron los niveles de eficiencia para un conjunto de Gobiernos locales en la República Checa para explorar la incidencia del grado de congruencia ideológica del alcalde dentro del consejo municipal en los niveles de eficiencia. Los resultados muestran que alcaldes con ideologías de izquierda son más ineficientes en su gestión municipal y que el grado de apoyo en el consejo municipal de este alcalde incrementa aún más esta ineficiencia. Para el caso chileno, pero en un contexto distinto, Chumacero y Paredes (2012) encuentran que la prevalencia de beneficiarios de programas de empleo está fuertemente vinculada a consideraciones políticas, y en menor medida a criterios asociados al nivel de desempleo y/o a la vulnerabilidad al desempleo o a la pobreza de la comuna. Lo anterior podría sugerir una incidencia de la política en la asignación eficiente de los recursos en los programas sociales en Chile. Nuestro trabajo contribuye a esta literatura entregando nueva evidencia para Chile sobre cómo afecta la relación entre la ideología política del Gobierno local con el Gobierno central en los niveles de eficiencia. Un elemento novel de nuestra muestra es que incorpora un período de información que nos permite explotar la variación existente desde dos elecciones, una de índole municipal en el año 2012 y otra presidencial en el año 2013, en las cuales se produce un cambio de Gobierno desde uno a otro sector político.

El artículo se estructura como sigue. En la sección 2 se presenta un conjunto de antecedentes que describen el funcionamiento, ingresos y gastos de las municipalidades en Chile. La sección 3 describe la metodología y el proceso de estimación en dos etapas. La sección 4 presenta los datos. La sección 5 discute los principales resultados. La sección 6 presenta un conjunto de test de robustez y la sección 7 concluye.

\section{Antecedentes de LAS MUNiCipalidades EN ChILE}

La figura de los Gobiernos locales en Chile se encuentra representada por las municipalidades, quienes según la legislación nacional ${ }^{1}$, tienen a su cargo la administración local de cada comuna o agrupación de comunas que determine la ley, agregando además que las municipalidades son corporaciones autónomas de derecho público, con personalidad jurídica y patrimonio propio. Su principal objetivo es satisfacer las necesidades de la comunidad local y asegurar su participación en el progreso económico, social y cultural. En cuanto a la dirección y gestión de las municipalidades, su máxima autoridad es el alcalde, que tiene a su cargo la dirección, administración superior y supervigilancia de su funcionamiento. Un

\footnotetext{
Este apartado se basa en lo establecido en el D.F.L 1-19.704 D.O. 03.05.2002 texto refundido, coordinado y sistematizado de la Ley $\mathrm{N}^{\circ} 18.695$, Orgánica Constitucional de Municipalidades de Chile.
} 
rol importante en la administración lo tiene el consejo municipal, el cual posee un carácter normativo, resolutivo y fiscalizador, encargado de hacer efectiva la participación de la comunidad local y de ejercer las atribuciones que le señale la ley. En el caso del alcalde, este es elegido por sufragio universal, en votación conjunta y cédula separada de la de los concejales, con un mandato de cuatro años con posibilidad de reelección. Por su parte, el consejo estará integrado por un grupo de concejales elegidos por votación directa mediante un sistema de representación proporcional. La duración es la misma que en el caso del alcalde, teniendo también la posibilidad de ser reelegidos.

Respecto a las funciones de las municipalidades, en primer lugar, se encuentran las que son ejercidas de manera privativa, es decir, solo por la municipalidad, como la planificación y regulación de la comuna así como la aplicación de las disposiciones tanto del transporte y tránsito público, construcción y urbanización, además de las responsabilidades del aseo y ornato de la comuna. En segundo lugar, se encuentran las que ejerce directamente o en conjunto con otros órganos de la administración del Estado, siendo estas las relacionadas con la educación y cultura, la salud pública, la asistencia social y jurídica, la capacitación y promoción del empleo, el turismo y deporte, la urbanización y vialidad, entre otras.

Para llevar a cabo el logro de sus objetivos, las municipalidades cuentan con recursos los cuales son generados ya sea de manera interna o externa. En el caso de los ingresos municipales, estos se encuentran principalmente divididos en tres grandes grupos: los Ingresos Propios Permanentes (IPP), el Fondo Común Municipal (FCM) y las Transferencias Gubernamentales (TG). Los Ingresos Propios Permanentes (IPP) son aquellos ingresos generados por recaudación de patentes municipales, por derechos de aseo, por concesiones, además de las rentas a la propiedad municipal y porcentajes del ingreso provenientes del impuesto territorial y de los permisos de circulación, también denominado certificado de matriculación del vehículo o certificado de registro del vehículo. Las fuentes que generan un mayor aporte al ingreso propio permanente son el impuesto territorial, las patentes comerciales y los permisos de circulación. Cabe destacar, que estos ingresos no se encuentran sujetos a restricciones para su inversión y son de libre disposición para el municipio correspondiente.

El Fondo Común Municipal (FCM) es un mecanismo de retribución solidaria de recursos financieros entre las distintas municipalidades del país. Dicho fondo se compone por un porcentaje aportado por cada municipalidad de los ingresos originados desde el impuesto territorial, ingresos por concepto de permisos de circulación de vehículos, ingresos por el pago de patentes y la Ley sobre expendio y consumo de bebidas alcohólicas, además de un 50\% del derecho de las transferencias de vehículos con permiso de circulación ${ }^{2}$. De igual modo se adhiere a este fondo, el monto total del impuesto territorial que paguen los inmuebles

$2 \quad$ Ley $\mathrm{N}^{\circ} 19.816$ Art. $^{\circ}$ a) D.O. 07.08.2002. Estos porcentajes varían para algunas comunas. En el caso de Las Condes, Providencia, Vitacura y Santiago, algunos de estos aportes tienden a ser mayores. 
fiscales afectos a dicho impuesto ${ }^{3}$ y un aporte fiscal considerado anualmente, a su valor del mes de agosto del año precedente. Por último, se incorpora un $100 \%$ de lo que se recaude por multas impuestas por el juzgado de policía local, por infracciones o contravenciones a las normas de tránsito ${ }^{4}$. Cabe destacar, que la distribución del Fondo está sujeta a las normas y criterios establecidos en la Ley modificada de rentas municipales ${ }^{5}$. De esta manera, un $25 \%$ del total es repartido en partes iguales entre todas las comunas, un $10 \%$ en relación con el grado de pobreza de la comuna ponderado con la población pobre del país, un $30 \%$ con base en la proporción de predios exentos de impuesto territorial de cada comuna y finalmente un $35 \%$ en proporción directa a los menores ingresos propios permanentes del año precedente al cálculo (Pérez, 2016).

Finalmente se debe mencionar las Transferencias Gubernamentales (TG), las cuales se encuentran representadas por aquellos recursos provenientes del Gobierno central y cuya finalidad es la de financiar directamente programas sectoriales y políticas públicas, tales como educación pública y salud primaria, además de vivienda, protección social, seguridad, urbanismo y otros proyectos de inversión obtenidos a través del (FNDR) Fondo Nacional de Desarrollo Regional (Henríquez \& Fuenzalida, 2011).

\section{Metodología}

La conceptualización del estudio de la eficiencia en la gestión de los gobiernos locales puede ser remontado al trabajo realizado por Renne (1937), quien planteó que la eficiencia puede ser entendida como la razón de salida a la entrada, por ejemplo, la cantidad de valor que es entregado en términos de servicio a cambio de un determinado gasto de dinero. No obstante, debido a los problemas de disponibilidad y calidad de información, los primeros intentos para medir la eficiencia estaban limitados a las comparaciones de gastos. No fue hasta los avances de tipo metodológico a finales de la década de los 70' e inicios de los 80', por medio del Análisis de Frontera Estocástica ${ }^{6}$ (SFA) y el Análisis Envolvente de Datos ${ }^{7}$ (DEA) en conjunto con mejoras en el registro de la información por parte de los gobiernos locales, que se comenzó a investigar de manera rigurosa la eficiencia en el área de la gestión pública, más concretamente en las unidades de Gobierno local o municipalidades. Si bien ambas técnicas permiten la medición del rendimiento, SFA como modelo estocástico, parte de la presunción de que la función de producción posee una determinada forma, y es capaz de diferenciar entre ineficiencia y ruido estadístico, adoptándose

\footnotetext{
Ley $\mathrm{N}^{\circ} 20.033$ Art. $5^{\circ} \mathrm{N}^{\circ} 2$ b) D.O. 01.07.2005.

Ley $\mathrm{N}^{\circ}$ 19.816 Art. $2^{\circ}$ c) D.O. 07.08.2002.

Ley 20.237 Art. $2^{\circ} \mathrm{N}^{\circ} 1$ D.O. 24.12.2007 y Ley 20.410 Art. 5 D.O. 20.01.2010.

Para más detalles revisar (Aigner, Lovell, \& Schmidt, 1977).

7 Para más detalles revisar (Banker et al., 1984; Charnes et al., 1978).
} 
principalmente en los campos de la investigación económica. En tanto, el DEA como un método no paramétrico o determinístico asume que la distancia de la unidad analizada a la frontera es fruto de la ineficiencia, y es concebido como una técnica estándar en la investigación operativa (Hollingsworth et al., 1999; Lampe \& Hilgers, 2015). En este trabajo, se propone la aplicación del modelo DEA en dos etapas. En la primera etapa, se utiliza un modelo DEA para calcular los puntajes de eficiencia de cada municipalidad. En una segunda etapa, con el fin de explorar e identificar las posibles causas de las diferencias en los niveles de eficiencia, se estima un modelo Probit Fraccional para datos de panel y un modelo de probabilidad lineal utilizando log odds ratios.

\subsection{Modelo DEA}

El Análisis Envolvente de Datos más conocido como DEA por la sigla de su nombre en inglés (Data Envelopment Analysis) fue desarrollado por Charnes et al. (1978) basados en el trabajo preliminar de Farrell (1957) y posteriormente ampliado por Banker, Charnes \& Cooper, (1984). DEA es un modelo no paramétrico que utiliza una técnica de programación lineal y permite evaluar la eficiencia relativa de un conjunto de unidades productivas. Este modelo no necesita la especificación de una forma funcional y su cálculo se realiza a través de la construcción de una frontera de producción, compuesta por aquellas unidades eficientes, que logran alcanzar el nivel máximo de outputs (salidas), utilizando un nivel determinado de inputs (entradas), esto en su versión orientada a los outputs. O bien, aquellas que logran un nivel determinado de outputs, utilizando la cantidad mínima de inputs, en el caso de su versión orientada a los inputs. Este estudio se enmarca en la orientación a los outputs, bajo el supuesto de que las municipalidades operan en base a un presupuesto anual, buscando idealmente maximizar la cantidad de servicios prestados a la comunidad de acuerdo a los recursos disponibles con los que cuentan. De esta manera, se puede determinar la ineficiencia de las unidades restantes ubicadas por debajo de la frontera, calculando la distancia a la que estas se encuentran con respecto a la frontera eficiente (Afonso \& Fernandes, 2006; Banker et al., 1984; Borger \& Kerstens, 1996; Charnes et al., 1978; Fernández Santos \& Flórez López, 2006; Worthington, 2000). En el lenguaje DEA, las municipalidades se denominan DMUs, cuya abreviación proviene de su definición en inglés, Decisión Making Unit, lo cual se traduce como Unidad Tomadora de Decisiones.

En este estudio se utilizó la versión del modelo DEA con retornos constantes a escala propuesto por Charnes et al. (1978), la cual es expresada como sigue:

$$
\max _{(u, v)} h_{0}=\frac{\sum_{r=1}^{s} u_{r} y_{r 0}}{\sum_{i=1}^{m} v_{i} x_{i 0}}
$$

Sujeto a las siguientes restricciones: 


$$
\begin{gathered}
\frac{\sum_{r=1}^{s} u_{r} y_{r 0}}{\sum_{i=1}^{m} v_{i} x_{i 0}} \leq 1 ; j=1 \ldots n \\
u_{r} v_{j} \geq 0 ; \quad r=1 \ldots \ldots s ; \quad i=1 \ldots \ldots m
\end{gathered}
$$

Donde en el numerador del cociente descrito en la ecuación (1) se encuentra la suma de $\mathrm{y}_{\mathrm{r} 0}$, que representa la cantidad de outputs $(1,2, \ldots \mathrm{r})$ producidos por la unidad evaluada representada por el subínidce 0 , multiplicados por los coeficientes de ponderación $\left(\mathrm{u}_{1}, \mathrm{u}_{2}, \ldots, \mathrm{u}_{\mathrm{r}}\right)$, que pueden ser considerados como un "precio" asociado a los outputs. Por otro lado, en el denominador se encuentra la suma de $\mathrm{x}_{\mathrm{i} 0}$, que representa la cantidad de inputs $(1,2, \ldots \mathrm{i})$ utilizados por la unidad evaluada en la producción de los outputs recogidos en el numerador, multiplicados por un coeficiente de ponderación $\left(\mathrm{v}_{1}, \mathrm{v}_{2} \ldots, \mathrm{v}_{\mathrm{i}}\right)$, que representan el "precio" asociado a cada input correspondiente, y que es distinto para cada unidad.

Así, el problema a resolver para cada DMU es encontrar el conjunto de "precios" $\left(\mathrm{u}_{\mathrm{r}}, \mathrm{v}_{\mathrm{i}}\right)$ que maximicen el valor del output de la unidad analizada con respecto al coste de sus inputs consumidos, resultando el ratio de eficiencia de cada DMU. A partir de las ponderaciones $\left(\mathrm{u}_{\mathrm{r}}, \mathrm{v}_{\mathrm{i}}\right)$ para cada unidad de producción, las restricciones descritas en las ecuaciones (2) y (3) pretenden asegurar que el cociente resultante de la ecuación (1) no sea superior a 1 para ninguna de las DMUs estudiadas. De forma esta forma, una DMU se considera eficiente cuando el resto de unidades no presentan una valoración superior a ella, alcanzando h0 el valor 1; siendo ineficientes aquellas otras DMUs que toman valores de $\mathrm{h}_{0}$ entre 0 y 1 .

Cabe agregar que, con el propósito de capturar los niveles de productividad y evolución de la eficiencia técnica en el tiempo, los cómputos de eficiencia se desarrollan dentro del índice de Fare-Primont (Färe \& Primont, 1995). Una de las ventajas de este índice es que supera las pruebas de transitividad, implicando consistencia para realizar comparaciones de productividad y eficiencia técnica multitemporales (O’Donnell, 2011).

\subsection{Modelos explicando los niveles de eficiencia}

Dado que las puntuaciones de eficiencia están típicamente definidas dentro del intervalo cero y 1 , con observaciones en los extremos reflejando soluciones de esquina, se propone la estimación de modelos de respuesta fraccional para datos de panel como sugerido por Papke \& Wooldridge (2008). Este modelo se estima por métodos de cuasi verosimilitud sobre la base de una distribución Bernoulli para asegurar que los valores predichos de eficiencia se encuentren entre cero y uno (Papke \& Wooldridge, 1996). El modelo se especifica a continuación:

$$
E\left(E T_{i t} \mid z_{i t}, c_{i}\right)=\Phi\left(z_{i t} \beta+c_{i}\right)
$$


Donde $E\left(E T_{i t} \mid z_{i t}, c_{i}\right)$ es el valor esperado de los puntajes de eficiencia para cada municipalidad $i$ y momento $t, \Phi$ denota una distribución normal acumulada estándar, $\beta$ son los parámetros estimados del modelo, $z_{i t}$ es un vector caracterizando un conjunto de variables explicativas en esta segunda etapa y $c_{i}$ corresponde a características no observadas específicas a cada comuna o municipalidad.

Para controlar por las características individuales no observadas, Wooldridge (2010) sugiere agregar al modelo el promedio de las variables que presentan variación a través del tiempo, controlando así, la heterogeneidad individual de éstas, lo cual se especifica de la siguiente manera:

$$
C_{i}=\psi+\varphi \overline{z_{i}}+a_{i}
$$

Donde $\overline{z_{i}}$ es un vector de los promedios de tiempo y $a_{i} \sim N\left(0, \sigma_{a}\right)$ es un residuo distribuido normal estándar. De este modo, al incluir la ecuación (5) en (6), el modelo queda especificado como sigue:

$$
E\left(E T_{i t} \mid z_{i t}, c_{i}, a_{i}\right)=G\left(\psi+z_{i t} \beta+\varphi \overline{z_{i}}+a_{i}\right)
$$

$0.25 \%$ por cada aumento en $1 \%$ en el porcentaje de consejeros de la misma coalición.

Uno de los cuestionamientos del modelo probit fraccional para datos de panel es que descansa en el supuesto de que la heterogeneidad no observada es condicional a los factores observados, y por lo tanto, independiente de otros factores excluidos del modelo. Alternativamente, se propone estimar un modelo de probabilidad lineal utilizando log-odds ratios, el cual asume que log-odds es una función lineal de los regresores, lo que permite agregar efectos fijos a nivel de municipalidades directamente a través de variables dummies. El modelo se especifica a continuación:

$$
\log \left(E T_{i t} 11-E T_{i t}\right)=z_{i t} \beta+D M_{i}+\varepsilon_{i t}
$$

Donde $\log \left(E T_{i t} 11-E T_{i t}\right)$ es el log-odds ratio de los puntajes de eficiencia para cada municipalidad $i$ y momento $t, \beta$ son los parámetros estimados del modelo, $z_{i t}$ es un vector caracterizando un conjunto de variables explicativas en esta segunda etapa, $D M_{i}$ denotan variables dummies a nivel de municipalidad, y $\varepsilon_{i t}$ corresponde al error del modelo. El modelo se estima vía mínimos cuadrados ordinarios (MCO)

\section{Datos}

Para el caso de las variables de la función de producción, se utilizan los indicadores de gestión municipal y sus Fichas Comunales de los años 2010 
al 2016 proporcionadas por el Servicio Nacional de Información Municipal (SINIM). Para las variables asociadas a factores políticos, se utiliza la información disponible desde el Servicio Electoral de Chile (SERVEL). Cabe destacar que, durante este período se observan elecciones de índole municipal en el 2012 y presidencial en el año 2013, que permiten identificar cambios de Gobierno de uno a otro sector político (ver Figura A2.1). Para controlar por el tamaño de las municipalidades, se utilizaron indicadores per cápita con estimaciones de población comunal para los años de interés calculadas por el Instituto Nacional de Estadística de Chile (INE). Para el caso indicadores de pobreza, se utilizó los datos de la encuesta CASEN correspondiente.

Aunque la literatura señala cierta subjetividad en el proceso de selección de variables, también se enfatiza que esta depende de la disponibilidad de datos (Cordero, et al., 2017; Díaz \& de Jorge, 2018). Si bien la selección de las variables en la primera etapa es restringida por la disponibilidad de información, las variables output e input consideradas en este estudio fueron seleccionadas siguiendo la literatura que se vincula a la eficiencia de los gobiernos municipales, tanto en la gestión de los recursos públicos (Díaz \& de Jorge, 2018; Silva \& Crisóstomo, 2019) como en los resultados de desempeño e indicadores multidimensionales de calidad de vida comunal (Silva et al., 2019).

$\mathrm{X}_{1}$ : Gasto total municipal por habitante (Gasto_Muni), variable cuantitativa que corresponde al total de los gastos ${ }^{8}$ efectuados por cada municipalidad durante cada año comprendido en la serie 2010-2016, y que fueron devengados al 31 de diciembre de cada año, dividido por la cantidad de habitantes estimada por el INE también para cada año, los cuales se encuentran expresados en miles de $\operatorname{pesos}^{9}(\mathrm{M} \$)$.

$\mathrm{X}_{2}$ : Número de funcionarios municipales por cada mil habitantes (Func_Muni), variable cuantitativa que corresponde a la suma total de funcionarios de planta y a contrata de cada municipalidad en cada año, divididos por la población estimada por el INE para cada año de la serie 2010-2016 y luego multiplicado por mil.

Estas dos primeras variables buscan representar los recursos con los que cuenta cada municipalidad para realizar su gestión.

$\mathrm{Y}_{1}$ : Porcentaje de Asistencia Escolar Comunal (Asist_Esc), variable de tipo cuantitativa que corresponde al porcentaje de asistencia escolar mensual promedio obtenido por los establecimientos educacionales municipales de cada comuna para cada año respectivo.

$\mathrm{Y}_{2}$ : Número de Consultas médicas realizadas en APS cada 1000 habitantes (Cons_APS), variable cuantitativa que mide el número total de consultas

8 Dentro de los cuales podemos encontrar: gastos en bienes y servicios de consumo, gasto en iniciativas de inversión, gastos en prestaciones de seguridad social, otros gastos corrientes, gasto en personal, gastos en adquisición de activos financieros y no financieros, préstamos, entre otros.

9 Valores a los cuales se les aplicó un factor de actualización monetaria, por lo que luego de dicha corrección, se despliegan valores en miles de pesos reales correspondientes al último mes de diciembre del año vigente publicado (Considera como año base el 2009). 
médicas realizadas en Atención Primaria de Salud (APS) en la comuna durante cada año, dividido por la cantidad de habitantes estimada por el INE para cada año y multiplicado por 1000 .

$\mathrm{Y}_{3}$ : Índice de superación de pobreza (Sup_Pobr), variable cuantitativa, correspondiente al porcentaje de habitantes que han superado la barrera de la pobreza, es decir, aquellos que se encuentran fuera del umbral de la pobreza, según la encuesta CASEN.

$\mathrm{Y}_{4}$ : Áreas verdes con mantenimiento por habitante (Area_Verd), variable de tipo cuantitativa que mide la cantidad de metros cuadrados de áreas verdes con mantenimiento por habitante, es decir, aquellos espacios con que cuentan las comunas, que son destinados al esparcimiento, recreación o tránsito peatonal de su población.

Estas últimas cuatro variables buscan representar los servicios prestados por las municipalidades a la comunidad a través de sus distintas áreas de gestión.

En la Tabla 1, se muestra un resumen descriptivo de las variables outputinput utilizadas en el modelo DEA.

En cuanto a la segunda etapa, la variable dependiente definida como el puntaje de Eficiencia Técnica Municipal $E T_{i t}$ obtenido para cada municipalidad incluida en la muestra para cada año respectivo, se relaciona con una serie de variables que buscan explorar la incidencia de variables de orden político en la eficiencia en la gestión municipal. De esta manera se incluye Cambio_Alcalde, variable dicotómica que captura el eventual cambio de alcalde en cada comuna luego de un proceso de elecciones municipales; Alcalde_Candidato, variable dicotómica que refleja si el alcalde de alguna comuna fue candidato en algún proceso de elecciones municipales; Alcalde_Concert, variable dicotómica que corresponde a la ideología política a la cual pertenece el alcalde de cada comuna, en este caso la Concertación; Concejales_Concert, variable que representa el porcentaje de concejales pertenecientes a la coalición de la Concertación respecto al total de concejales; Gobierno_Concert, variable de tipo dicotómica que corresponde a la ideología política a la cual pertenece el gobierno de turno, en este caso toma valores de 1 si el presidente es de la coalición de Concertación y 0 si es de otra coalición.

TABLA 1

ESTADÍSTICA DESCRIPTIVA VARIABLES MODELO DEA

\begin{tabular}{|lrrrr|}
\hline Variable & Máximo & Mínimo & Media & Desv. Est. \\
\hline Gasto_Muni & 11976,08 & 20,27 & 310,68 & 597,79 \\
Func_Muni & 128,44 & 0,24 & 3,71 & 5,63 \\
Asist_Esc & 100 & 0 & 87,66 & 4,82 \\
Cons_APS & 6259,65 & 0 & 1062,2 & 647,42 \\
Sup_Pobr & 99,89 & 42,95 & 81,67 & 8,94 \\
Area_Verd & 71,9 & 0 & 3,83 & 4,84 \\
\hline
\end{tabular}

Fuente: elaboración propia. 
Se debe agregar que para la construcción de estas tres últimas variables se consideró que desde el año 2010 en adelante, los partidos pertenecientes a la Concertación en Chile fueron: Democracia Cristiana, Partido por la Democracia, Partido Socialista y Partido Radical Socialdemócrata. Luego de lo cual, a partir del año 2013 en adelante dicha coalición pasa a denominarse Nueva Mayoría conformada por los partidos de: Democracia Cristiana, Partido por la Democracia, Partido Socialista, Partido Radical Socialdemócrata, Partido Izquierda Ciudadana, Partido Comunista y Partido Movimiento Amplio Social, con lo cual para uso del estudio se consideró opositor a cualquier otro partido o coalición distinta a los aquí mencionados.

El principal objetivo de este estudio es explorar si las municipalidades administradas por un Gobierno local de la misma coalición política a la del Gobierno central gestionan más eficientemente sus recursos. La coalición del Gobierno local se aproxima a través de la identificación de la coalición del alcalde en ejercicio o el porcentaje de consejeros municipales de la misma coalición. Para responder al objetivo, estas variables se interactúan con la identificación de la coalición del Gobierno central. La identificación proviene desde la temporalidad de la información, la cual comprende dos elecciones, una de índole municipal en el año 2012 y otra presidencial en el año 2013, en las cuales se observan cambios en las coaliciones que gobiernan las municipalidades y el país (ver Figura A1).

En las estimaciones controlamos por una serie de variables a nivel municipal: (Población), variable que representa el total de la población para cada comuna estimados por el INE (Instituto Nacional de Estadística); Part_IPP, variable que representa el porcentaje que poseen los ingresos propios permanentes percibidos por cada municipalidad, respecto del total de sus ingresos del presupuesto para cada año, intentando reflejar con esto el grado de autonomía para generar sus ingresos; Part_FCM, variable que indica el porcentaje que representan los ingresos provenientes del fondo común municipal, respecto del total de los ingresos percibidos por cada municipalidad. Población_Rural, variable que corresponde al porcentaje de población rural que posee la comuna respecto al total de población comunal. ${ }^{10}$ Porcentaje de Funcionarios Profesionales (Func_Profesional), variable que corresponde a la proporción de funcionarios municipales que poseen la calificación de profesionales, respecto del total de funcionarios municipales en cada comuna, considerando funcionarios de planta y a contrata de cada municipalidad.

La Tabla 2 resume las estadísticas descriptivas de las variables de la segunda etapa.

10 Se entiende por población rural a todos aquellos asentamientos humanos, concentrados o dispersos, cuya población no supera los 1.000 habitantes o entre 1.001 y 2.000 en caso de que la población económicamente activa dedicada a las actividades secundarias y/o terciarias sea inferior al 50\%. Esta variable fue elaborada con datos proveniente de las proyecciones de población con los datos demográficos observados hasta el año 2002 por el INE, donde se consideró como año base el 2010. 
TABLA 2

ESTADÍSTICA DESCRIPTIVA VARIABLES MODELO PROBIT FRACCIONAL

\begin{tabular}{|lrrrr|}
\hline Variable & Máximo & Mínimo & Media & Desv. Est. \\
\hline ET & 1,00 & 0,01 & 0,51 & 0,22 \\
Población & 931,21 & 0,10 & 57,46 & 94,74 \\
Part_IPP & 82,12 & 2,01 & 26,23 & 16,47 \\
Part_FCM & 87,87 & 1,14 & 44,41 & 17,91 \\
Población_Rural & 100,00 & 0,00 & 37,20 & 28,94 \\
Func_Profesional & 100,00 & 0,00 & 27,42 & 9,54 \\
Cambio_Alcalde & 1,00 & 0,00 & 0,27 & 0,44 \\
Alcalde_Candidato & 1,00 & 0,00 & 0,21 & 0,41 \\
Alcalde_Concert & 1,00 & 0,00 & 0,30 & 0,46 \\
Concejales_Concert & 66,67 & 0,00 & 30,86 & 12,19 \\
Gobierno_Concert & 1,00 & 0,00 & 0,43 & 0,50 \\
\hline
\end{tabular}

Fuente: elaboración propia.

\section{Resultados}

\subsection{Puntajes de eficiencia y dinámica temporal}

Las estadísticas descriptivas de los puntajes de eficiencia obtenidas en la primera etapa se muestran en la Tabla 3. Los resultados arrojan un nivel promedio de eficiencia técnica para el periodo 2010-2016 de un 51\%, lo que implica que en promedio las municipalidades proveer un $49 \%$ menos de bienes y servicios que

TABLA 3

RESUMEN ESTADÍSTICO PUNTAJES DE EFICIENCIA TÉCNICA MUNICIPAL

\begin{tabular}{|ccccc|}
\hline Periodo & Máximo & Mínimo & Media & Desv. Est. \\
\hline 2010 & $100 \%$ & $1,84 \%$ & $51,15 \%$ & $20,45 \%$ \\
2011 & $100 \%$ & $1,57 \%$ & $51,53 \%$ & $21,14 \%$ \\
2012 & $100 \%$ & $2,08 \%$ & $52,83 \%$ & $22,57 \%$ \\
2013 & $100 \%$ & $1,48 \%$ & $52,12 \%$ & $22,42 \%$ \\
2014 & $100 \%$ & $1,03 \%$ & $53,05 \%$ & $21,85 \%$ \\
2015 & $100 \%$ & $1,67 \%$ & $50,70 \%$ & $21,74 \%$ \\
2016 & $100 \%$ & $1,13 \%$ & $43,76 \%$ & $21,05 \%$ \\
Total & $100 \%$ & $1,03 \%$ & $50,74 \%$ & $21,78 \%$ \\
\hline
\end{tabular}

Fuente: elaboración propia a partir de los resultados. 
en una situación óptima. Además, se observa que el 93\% de las municipalidades poseen puntajes de eficiencia menores a un $80 \%$ y el $69 \%$ de ellas no supera un $50 \%$ en sus puntuaciones. Por otra parte, al observar los puntajes de eficiencia técnica promedio por año, se puede observar una reducción en la eficiencia técnica promedio que va desde un 51\% en el año 2010 a un 44\% en el año 2016.

La Figura 1 muestra los promedios de eficiencia para las municipalidades utilizadas en este estudio. El mapa revela una gran heterogeneidad en el desempeño municipal con relación al uso de recursos a lo largo del país, con niveles

FIGURA 1

PUNTAJES PROMEDIO EFICIENCIA TÉCNICA POR COMUNA A LO LARGO DEL PAÍS
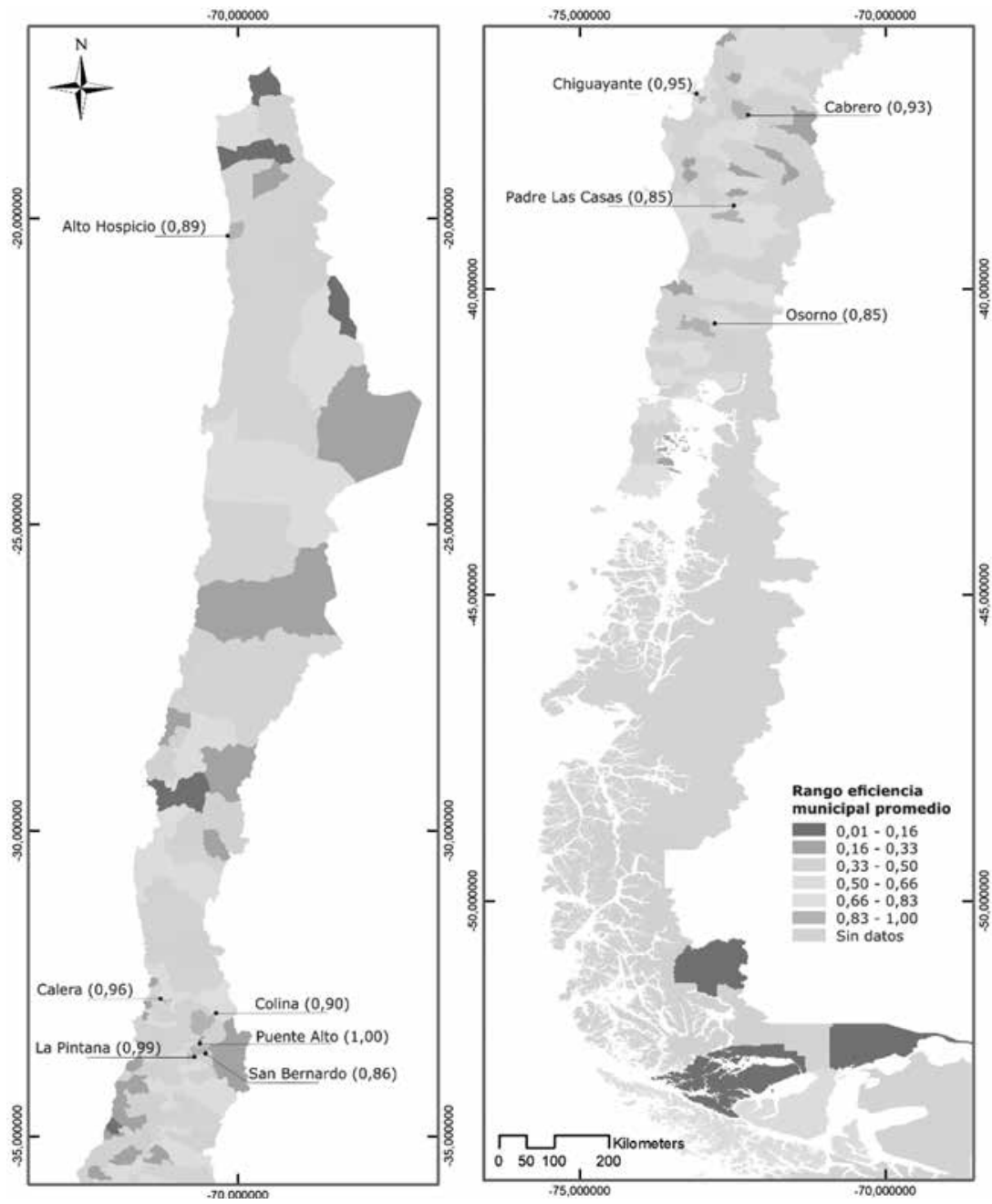

Fuente: elaboración propia a partir de los resultados. 
promedio de eficiencia más altos en la zona centro-sur y desempeños más bajos observados en los extremos norte y sur del país.

\subsection{Determinantes de la eficiencia comunal}

En relación a los factores que indicen en la eficiencia municipal, la Tabla 4 muestra los resultados tras estimar el modelo Probit Fraccional.

Respecto a las variables de control, el coeficiente asociado a la Población Comunal (Población) resultó ser positivo y significativo al 1\%, implicando que una mayor población comunal influiría de forma positiva en el nivel de eficiencia obtenido por la municipalidad. Esto podría estar relacionado a que municipalidades de mayor tamaño podrían aprovechar economías de escala para ser más eficientes. Asimismo, un mayor número de demandantes de servicios municipales ejercería una mayor presión para que la municipalidad gestione de mejor manera y de forma más eficiente sus recursos con el objetivo de cubrir las necesidades de sus habitantes. Los resultados también muestran que una mayor participación del Fondo Común Municipal en el ingreso total (Part_FCM) influye positivamente y significativamente en la eficiencia municipal. Esto puede deberse a que las municipalidades que reciben una mayor proporción de sus ingresos de este tipo de fuentes se encuentran menos restringidas en el uso que le dan a dichos recursos. Lo anterior es en términos relativos a las que reciben mayores porcentajes de recursos provenientes de las transferencias gubernamentales, los cuales en su mayoría deben por mandato o ley ser utilizados en los ítems específicos a los cuales van dirigidos. Por otro lado, el coeficiente de Población Rural Comunal (Población_Rural) resultó significativo al 1\% pero con signo negativo, lo que implica que a mayor grado de ruralidad en la comuna, menor será el nivel de eficiencia alcanzado. Las comunas rurales se caracterizan por presentar distancias más largas de recorrido, menor acceso a tecnologías y menor accesibilidad por parte de las personas a los servicios requeridos, lo que dificulta de esta manera el desarrollo de la gestión de los municipios en la cobertura y prestación de los servicios municipales. Cuando se controla mediante dummies por años, el coeficiente de participación de los Ingresos Propios Permanentes en el ingreso total (Part_IPP) resulta ser significativo al 10\% con signo positivo, lo que quiere decir que una mayor Participación de los Ingresos Propios Permanente en el Ingreso Total, influirá de forma positiva en el nivel de eficiencia obtenido por la municipalidad. Los Ingresos propios permanentes son una fuente de recursos sobre la cual las municipalidades poseen una mayor autonomía en cuanto a su utilización, pudiendo ser destinados según la eventual necesidad del municipio en cuestión.

Desde la columna (3) en delante se adicionan al modelo las variables de orden político. La última columna controla por heterogeneidad individual. De esta manera, controlando por dummies por año los resultados muestran significancia estadística al 5\% para la variable Alcalde Candidato (Alcalde_Candidato), implicando que los alcaldes en ejercicio que asumen una candidatura municipal para el próximo periodo tienden a ser más eficientes en la gestión de su municipio. 
TABLA 4

RESULTADOS MODELO PROBIT FRACCIONAL

\begin{tabular}{|c|c|c|c|c|c|c|}
\hline \multirow{2}{*}{ VARIABLES } & (1) & (2) & (3) & (4) & (5) & (6) \\
\hline & ET & ET & ET & ET & ET & ET \\
\hline \multirow[t]{2}{*}{ Población } & $0.00240 * * *$ & $0.00245^{* * *}$ & $0.00244^{* * *}$ & $0.00242^{* * *}$ & $0.00242^{* * *}$ & $0.00473^{* * *}$ \\
\hline & $(0.000604)$ & $(0.000613)$ & $(0.000626)$ & $(0.000624)$ & $(0.000624)$ & $(0.00129)$ \\
\hline \multirow[t]{2}{*}{ Part_IPP } & 0.00426 & $0.00500^{*}$ & $0.00587 * *$ & $0.00580^{* *}$ & $0.00579 * *$ & $0.00838 * * *$ \\
\hline & $(0.00277)$ & $(0.00282)$ & $(0.00273)$ & $(0.00271)$ & $(0.00271)$ & $(0.00190)$ \\
\hline \multirow[t]{2}{*}{ Part_FCM } & $0.0120 * * *$ & $0.0128 * * *$ & $0.0132 * * *$ & $0.0132 * * *$ & $0.0132 * * *$ & $0.0113^{* * *}$ \\
\hline & $(0.00213)$ & $(0.00218)$ & $(0.00206)$ & $(0.00207)$ & $(0.00207)$ & $(0.00140)$ \\
\hline \multirow[t]{2}{*}{ Población_Rural } & $-0.00656^{* * *}$ & $-0.00652 * * *$ & $-0.00637 * * *$ & $-0.00640 * * *$ & $-0.00640^{* * *}$ & $-0.00638 * * *$ \\
\hline & $(0.00148)$ & $(0.00149)$ & $(0.00143)$ & $(0.00144)$ & $(0.00144)$ & $(0.00148)$ \\
\hline \multirow[t]{2}{*}{ Func_Profesional } & -0.000825 & -0.00102 & -0.000730 & -0.000737 & -0.000759 & $-0.00158 * *$ \\
\hline & $(0.00123)$ & $(0.00122)$ & $(0.00120)$ & $(0.00120)$ & $(0.00122)$ & $(0.000664)$ \\
\hline \multirow[t]{2}{*}{ Cambio_Alcalde } & & & -0.0677 & -0.0641 & -0.0643 & -0.0679 \\
\hline & & & $(0.0565)$ & $(0.0569)$ & $(0.0570)$ & $(0.0559)$ \\
\hline \multirow[t]{2}{*}{ Alcalde_Candidato } & & & $0.0907 *$ & $0.0860^{*}$ & $0.0864^{*}$ & $0.0917 *$ \\
\hline & & & $(0.0500)$ & $(0.0503)$ & $(0.0502)$ & $(0.0496)$ \\
\hline \multirow[t]{2}{*}{ Alcalde_Concert } & & & 0.0372 & -0.0129 & -0.0441 & -0.0679 \\
\hline & & & $(0.0484)$ & $(0.165)$ & $(0.173)$ & $(0.173)$ \\
\hline \multirow[t]{2}{*}{ Concejales_Concert } & & & $0.00459^{* *}$ & 0.00209 & 0.00184 & 0.00165 \\
\hline & & & $(0.00203)$ & $(0.00251)$ & $(0.00257)$ & $(0.00253)$ \\
\hline \multirow[t]{2}{*}{ Gobierno_Concert } & & & $-0.248^{* * *}$ & $-0.371 * * *$ & $-0.388 * * *$ & $-0.407 * * *$ \\
\hline & & & $(0.0453)$ & $(0.0832)$ & $(0.0888)$ & $(0.0888)$ \\
\hline \multirow{2}{*}{$\begin{array}{l}\text { Concejales_Concert*Alcalde_ } \\
\text { Concert }\end{array}$} & & & & 0.00198 & 0.00288 & 0.00350 \\
\hline & & & & $(0.00450)$ & $(0.00476)$ & $(0.00478)$ \\
\hline \multirow{2}{*}{$\begin{array}{l}\text { Alcalde_Concert*Gobierno_ } \\
\text { Concert }\end{array}$} & & & & -0.0427 & 0.0247 & 0.0592 \\
\hline & & & & $(0.0571)$ & $(0.176)$ & $(0.173)$ \\
\hline \multirow{2}{*}{$\begin{array}{l}\text { Concejales_} \\
\text { Concert*Gobierno_Concert }\end{array}$} & & & & $0.00444 * *$ & $0.00501^{* *}$ & $0.00533 * *$ \\
\hline & & & & $(0.00207)$ & $(0.00243)$ & $(0.00241)$ \\
\hline \multirow{2}{*}{$\begin{array}{l}\text { Alcalde_Concert*Concejales_ } \\
\text { Concert*Gobierno_Concert }\end{array}$} & & & & & -0.00199 & -0.00272 \\
\hline & & & & & $(0.00511)$ & $(0.00501)$ \\
\hline \multirow[t]{2}{*}{ Constant } & $-0.489 * * *$ & $-0.521 * * *$ & $-0.733^{* * *}$ & $-0.656 * * *$ & $-0.648 * * *$ & $-0.832^{* *}$ \\
\hline & $(0.178)$ & $(0.179)$ & $(0.176)$ & $(0.181)$ & $(0.185)$ & $(0.386)$ \\
\hline Dummy por año & No & $\mathrm{Si}$ & $\mathrm{Si}$ & $\mathrm{Si}$ & $\mathrm{Si}$ & $\mathrm{Si}$ \\
\hline Log seudo verosimilitud & -758.7690 & -754.6711 & -752.1308 & -751.7446 & -751.7313 & -751.2162 \\
\hline Heterogeneidad individual & No & No & No & No & No & $\mathrm{Si}$ \\
\hline Observaciones & 1,631 & 1,631 & 1,631 & 1,631 & 1,631 & 1,631 \\
\hline
\end{tabular}

Los errores estándar robustos están entre paréntesis $* * * \mathrm{p}<0.01, * * \mathrm{p}<0.05,{ }^{*} \mathrm{p}<0.1$

Fuente: elaboración propia a partir de los resultados. 
Un alcalde con intereses políticos futuros se siente con más motivación para gestionar los recursos de forma más eficiente y con una visión y objetivos de más largo plazo, esperando con ello, la aprobación de los votantes y una futura reelección y continuidad en el cargo. Otra variable que resulta ser significativa es Gobierno de Concertación (Gobierno_Concert), sugiriendo que los municipios en periodos presidenciales gobernados por la Concertación (Nueva Mayoría), resultarían ser menos eficientes. Este resultado podría estar en línea con lo encontrado en la literatura con relación a que los Gobiernos dirigidos por políticos con ideología de izquierda tienden a ser menos eficientes (Št'astná \& Gregor, 2015). Lo anterior se sustenta en el hecho de que políticos de izquierda tienden a ponderar más objetivos de orden social relativamente a objetivos de índole económico, lo cual implica gastos más elevados para lograr mayor cobertura de servicios y asistencia, descuidando de esta manera la eficiencia en el gasto.

Adicionalmente, con el objeto de identificar de qué manera la relación entre el Gobierno local y nacional influyen en la eficiencia de los Gobiernos locales, se incluyen interacciones entre las variables Concejales de Concertación con Alcalde de Concertación, Alcalde de Concertación con Gobierno de Concertación, Concejales de Concertación con Gobierno de Concertación y Alcalde de Concertación con Concejales de Concertación y con Gobierno de Concertación. Los resultados muestran significancia estadística para la variable interactuada Concejales de Concertación con Gobierno de Concertación (Concejales_Concert*Gobierno_Concert), con signo positivo. De esta manera, los resultados sugieren que en presencia de Gobiernos de Concertación con ideología de izquierda, consistentes con menores niveles de eficiencia, un mayor número de Concejales de la misma coalición reduciría este efecto negativo. Lo anterior sugiere que al existir mayor presencia de concejales de Concertación, podría haber mayores y mejores esfuerzos de coordinación y articulación entre los objetivos del Gobierno central y los Gobiernos locales, lo que beneficia de esta manera la capacidad de gestión del municipio. Este resultado es consistente con lo encontrado por Acuña et al. (2019), quienes estiman una brecha en gasto social menor entre coaliciones de centro-izquierda y centro-derecha, pero aún a favor de la coalición de centro-izquierda, cuando los alcaldes de la coalición respectiva están alineados políticamente con el Gobierno central. Esto podría señalizar mayor eficiencia en gasto social.

Finalmente, se discute la importancia económica relativa de las variables de política observando la magnitud estimada de los efectos marginales (ver Tabla A1). Las comparaciones de estos efectos entre las distintas variables nos dan cuenta de una importancia relativa mayor de aquellas variables que miden la estructura de fuentes de financiamiento en relación con las de orden político. Por ejemplo, por cada aumento porcentual en la participación de los ingresos dependientes del fondo municipal, los niveles de eficiencia aumentarían un 0,19\%. Por otro lado, en promedio, las municipalidades en Gobiernos de coalición de la Concertación rinden un $0,06 \%$ menos de eficiencia, valor que se ve aminorado en un $0,025 \%$ por cada aumento en $1 \%$ en el porcentaje de consejeros de la misma coalición. 
Alternativamente, y considerando que el modelo probit fraccional no controla completamente por efectos fijos a nivel de municipalidades, y que el supuesto que utiliza para capturar la heterogeneidad no observada es cuestionable cuando asume que el error es independiente de todo lo demás, se estima un modelo de probabilidad lineal utilizando log-odds ratios, el cual permite agregar efectos fijos a nivel de municipalidades. Las estimaciones se presentan en la Tabla 5. Los

TABLA 5

RESULTADOS MODELO LOG-ODDS RATIO CON EFECTOS FIJOS POR MUNICIPALIDAD

\begin{tabular}{|c|c|c|c|c|c|}
\hline VARIABLES & ET & ET & ET & ET & ET \\
\hline Población & $0.003 * * *$ & $0.003 * * *$ & $0.00389 * * *$ & $0.00352 * * *$ & $0.00350 * * *$ \\
\hline Part_IPP & $\begin{array}{l}(0.000929) \\
0.0117^{* * *}\end{array}$ & $\begin{array}{l}(0.000797) \\
0.0128 * * *\end{array}$ & $\begin{array}{l}(0.000 / 98) \\
0.0124^{* * *}\end{array}$ & $0.0118^{* * *}$ & $0.0121 * * *$ \\
\hline & $(0.00287)$ & $(0.00262)$ & $(0.00264)$ & $(0.00264)$ & $(0.00266)$ \\
\hline Part_FCM & $\begin{array}{c}0.0139 * * * \\
(0.0022)\end{array}$ & $\begin{array}{l}0.018^{* * *} \\
(0.00227)\end{array}$ & $\begin{array}{c}0.0181 * * * \\
(0.00228)\end{array}$ & $\begin{array}{c}0.0184 * * * \\
(0.00225)\end{array}$ & $\begin{array}{c}0.0182 * * * \\
(0.00224)\end{array}$ \\
\hline Población_Rural & $\begin{array}{l}-0.019 * * * \\
(0.00343)\end{array}$ & $\begin{array}{c}-0.020^{* * *} \\
(0.0034)\end{array}$ & $\begin{array}{c}-0.0205^{* * *} * \\
(0.00341)\end{array}$ & $\begin{array}{c}-0.0214 * * * \\
(0.00349)\end{array}$ & $\begin{array}{c}-0.0211 * * * \\
(0.00352)\end{array}$ \\
\hline Func_Profesional & $\begin{array}{r}-0.00208 \\
(0.00129)\end{array}$ & $\begin{array}{c}-0.0024^{* *} \\
(0.00119)\end{array}$ & $\begin{array}{c}-0.00253^{* *} \\
(0.00119)\end{array}$ & $\begin{array}{c}-0.00263^{* *} \\
(0.00119)\end{array}$ & $\begin{array}{c}-0.00279 * * \\
(0.00119)\end{array}$ \\
\hline Cambio_Alcalde & & & $\begin{array}{c}0.000520 \\
(0.0475)\end{array}$ & $\begin{array}{c}0.0106 \\
(0.0471)\end{array}$ & $\begin{array}{c}0.0136 \\
(0.0471)\end{array}$ \\
\hline Alcalde_Candidato & & & $\begin{array}{l}-0.0375 \\
(0.0712)\end{array}$ & $\begin{array}{l}-0.0418 \\
(0.0702)\end{array}$ & $\begin{array}{c}-0.0401 \\
(0.0693)\end{array}$ \\
\hline Alcalde_Concert & & & $\begin{array}{r}-0.108 * * \\
(0.0458)\end{array}$ & $\begin{array}{c}-0.346^{* *} \\
(0.136)\end{array}$ & $\begin{array}{c}-0.489 * * * \\
(0.161)\end{array}$ \\
\hline Consejales_Concert & & & $\begin{array}{l}0.000559 \\
(0.00168)\end{array}$ & $\begin{array}{l}-0.00193 \\
(0.00206)\end{array}$ & $\begin{array}{r}-0.00300 \\
(0.00207)\end{array}$ \\
\hline Gobierno_Concert & & & $\begin{array}{c}-0.348 * * * \\
(0.0653)\end{array}$ & $\begin{array}{c}-0.310 * * * \\
(0.0950)\end{array}$ & $\begin{array}{c}-0.387 * * * \\
(0.0904)\end{array}$ \\
\hline $\begin{array}{l}\text { Consejales_Concert*Alcalde_ } \\
\text { Concert }\end{array}$ & & & & $0.00838 * *$ & $0.0124 * * *$ \\
\hline & & & & $(0.00358)$ & $(0.00437)$ \\
\hline Alcalde_Concert*Gobierno_ & & & & $-0.168^{* * *}$ & 0.157 \\
\hline Conce & & & & $(0.0584)$ & $(0.199)$ \\
\hline Consejales_Concert*Gobierno_ & & & & 0.000312 & 0.00301 \\
\hline Concer & & & & $(0.00216)$ & $(0.00231)$ \\
\hline Alcalde_Concert*Consejales_ & & & & & -0.00977 \\
\hline & & & & & $(0.00596)$ \\
\hline Constant & $\begin{array}{c}-1.582 * * * \\
(0.289)\end{array}$ & $\begin{array}{c}-1.765^{* * *} \\
(0.289)\end{array}$ & $\begin{array}{c}-1.748 * * * \\
(0.289)\end{array}$ & $\begin{array}{c}-1.623 * * * \\
(0.287)\end{array}$ & $\begin{array}{c}-1.601 * * * \\
(0.294)\end{array}$ \\
\hline Efectos fijos por año & No & $\mathrm{Si}$ & $\mathrm{Si}$ & $\mathrm{Si}$ & $\mathrm{Si}$ \\
\hline Efectos fijos municipalidades & $\mathrm{Si}$ & $\mathrm{Si}$ & $\mathrm{Si}$ & $\mathrm{Si}$ & $\mathrm{Si}$ \\
\hline R2 & 0.820 & 0.842 & 0.843 & 0.845 & 0.845 \\
\hline Observaciones & 1,580 & 1,580 & 1,580 & 1,580 & 1,580 \\
\hline
\end{tabular}

Los errores estándar robustos están entre paréntesis $* * * \mathrm{p}<0.01, * * \mathrm{p}<0.05, * \mathrm{p}<0.1$

Fuente: elaboración propia a partir de los resultados. 
resultados se mantienen en línea con los anteriores, revelando un efecto positivo de la variable que interactúa el porcentaje de concejales de la Concertación con la existencia de un Gobierno local de la misma coalición.

\section{Test de robustez}

Uno de los supuestos principales que se asume en la estimación de una frontera de producción a través del modelo DEA es que existen rendimientos constantes de escala. Sin embargo, las municipalidades podrían presentar economías de escala en la medida que la provisión de sus servicios alcance a una población más grande. Si esto es cierto, el supuesto puede ser incorrecto. Para explorar si los resultados cambian ante el incumplimiento de este supuesto, se estima un modelo DEA con rendimientos crecientes a escala. Los resultados son robustos a la estimación de puntuaciones de eficiencia bajo el supuesto de retornos variables de escala (ver Tabla A2).

Uno de los cuestionamientos del modelo DEA radica en el hecho de asumir una ecuación independiente de la frontera de producción para explicar los niveles de eficiencia técnica. El enfoque de fronteras estocásticas propone una estimación conjunta entre los determinantes de la frontera de producción y los niveles de eficiencia. Como un test de robustez, y dada la naturaleza multiproducto en la gestión de los Gobiernos locales, se estima una frontera estocástica de costos donde los gastos totales municipales son una función de los productos u output objetivos (ver Tabla A3). Una frontera de costos fue sugerida anteriormente por Pacheco et al. (2014) para el caso chileno. En esta estimación, se sigue el modelo propuesto por Greene (2005 $)$ y Greene (2005b), el cual aplica elementos del modelo de efectos fijos al enfoque de fronteras estocásticas. Los resultados no cambian fundamentalmente. La mayoría de los factores, explicando los valores de eficiencia, mantienen su signo y significancia, particularmente la variable interacción entre la proporción de concejales de la Concertación y la presencia de un Gobierno de la Concertación. En este caso, se obtiene un signo negativo, implicando que la ineficiencia de los Gobiernos locales disminuye en períodos presidenciales de la Concertación cuando la proporción de concejales de la misma coalición es mayor.

Finalmente, de acuerdo a la elección de los outputs para estimar la eficiencia en la gestión municipal, la pobreza aparece como una variable que podría estar relacionada con las preferencias políticas del electorado, en el sentido de que coaliciones de izquierda tienden a ser elegidas en municipalidades más pobres. De esta manera, podría haber una relación entre eficiencia y coaliciones de izquierda que no necesariamente significa que los alcaldes de izquierda son peores administradores. Para evaluar si este puede ser un problema, se estima la frontera de producción y el modelo explicando las eficiencias sin incluir la variable pobreza (ver Tabla A4). Los resultados principales se mantienen ante esta nueva especificación. Alternativamente, presentamos un test de exogeneidad de la variable afiliación política. Para tal efecto, estimamos un modelo de regresión lineal con efectos fijos, y realizamos un test de exogeneidad débil 
para las variables pertenencia del alcalde a la Concertación (Alcalde_Concert) y el porcentaje de concejales de la Concertación (Concejales_Concert), utilizando como instrumento la variable Superación de la pobreza (Sup_Pobr). Bajo heterocedasticidad condicional, este test de endogeneidad es numéricamente igual a un test estadístico de Hausman (Hayashi, 2000). Este test estadístico es distribuido chi-cuadrado con un número de grados de libertad igual al número de regresores evaluados. La hipótesis nula afirma que la variable es exógena. El estadístico Chi-cuadrado calculado fue de 0,364 (valor $\mathrm{p}=0,54$ ), lo que nos lleva a no rechazar la hipótesis nula, concluyendo que la afiliación política puede ser considerada como exógena.

\section{Conclusiones}

Los Gobiernos locales representan la figura de Gobierno más próxima a los ciudadanos, cumpliendo un rol fundamental en la entrega de servicios y la implementación de políticas públicas que satisfagan las demandas y requerimientos de la comunidad. Son también los principales responsables de administrar los recursos públicos, lo cual requiere que a la hora de cumplir su rol y utilizar dichos recursos, lo hagan de la manera más eficiente posible, adaptándose a las necesidades y preferencias de la comunidad.

Las estimaciones de eficiencia técnica de las municipalidades reportan una tendencia a la baja en el período bajo estudio, lo cual no deja de ser alarmante para la administración pública local. Los resultados muestran una gran heterogeneidad en los niveles de eficiencia de los Gobiernos locales en la gestión de recursos, con municipios logrando mantenerse cerca de la frontera eficiente por varios años, reportando valores promedio de un $96 \%$, comparados con otras comunas de muy bajo desempeño con puntajes promedio entre un $2 \%-6 \%$.

En relación a los factores que afectan los niveles de eficiencia, los resultados señalan que comunas más grandes, urbanas y con una mayor participación del Fondo Común Municipal e Ingresos Permanentes en su ingreso total presentan niveles de eficiencia más altos. Con respecto a los factores políticos, los resultados sugieren que en comunas donde el alcalde en ejercicio se presenta a reelección, las municipalidades tienden a ser más eficientes, mientras que en períodos en que gobierna una coalición de izquierda, la eficiencia promedio municipal se reduce. Esta reducción general en la eficiencia en Gobiernos de coaliciones de izquierda se ve atenuada cuando el grado de influencia de la misma coalición en el consejo municipal es mayor. Lo anterior soporta la hipótesis de la existencia de una relación entre los Gobiernos locales y el Gobierno central que incide en la manera como se gestionan los recursos municipales. Este trabajo sugiere que municipalidades con un mayor grado de apoyo en el consejo en línea con la ideología política del Gobierno local y central se encuentran en una mejor posición para gestionar sus recursos más eficientemente. Una de las posibles explicaciones se relaciona con mayores ventajas y facilidades que surgen en esta relación que pueden propiciar una mejor coordinación y articulación entre 
los objetivos del Gobierno central y los gobiernos locales, beneficiando de esta manera la capacidad de gestión del municipio. Estos resultados son robustos al supuesto de rendimientos variables de escala, a la estimación de una frontera estocástica de costos, a la exclusión de la variable pobreza del set de outputs, y la consideración de efectos fijos por municipalidades dentro de un modelo de probabilidad lineal utilizando log-odds ratios.

Cabe mencionar algunas limitaciones de este artículo. En primer lugar, se debe notar que, la estimación de la eficiencia técnica de las municipalidades mide la capacidad de gestión en cuanto a la entrega de servicios a la comunidad, es decir, una medición en términos de cobertura de servicios públicos, no así la calidad de este tipo de servicios prestados. En segundo lugar, las puntuaciones de eficiencia se expresan en términos relativos, debido a que se mide comparativamente solo a las unidades presentes en la muestra dentro de los periodos considerados en el análisis, por tanto, los puntajes de eficiencia técnica obtenidos para cada municipalidad presentan sensibilidad frente a eventuales cambios o modificaciones en la muestra. En tercer lugar, limitación de información impiden realizar una evaluación formal de la dirección de causalidad entre eficiencia y afiliación política. Sin embargo, los resultados excluyendo la variable pobreza de la frontera de producción entregan evidencia de exogenidad al menos en esta dimensión.

A pesar de estas limitaciones, desde este trabajo emergen importantes implicancias de política. Los resultados ratifican la importancia de la autonomía municipal, principalmente en lo referente a la generación de ingresos propios, ya sea por medio de la gestión de sus propios recursos o bien a través de la recaudación de algún tipo de impuesto. Lo anterior con la finalidad de equiparar en parte la desigualdad presente entre distintos municipios y disminuir la gran dependencia que poseen algunos de ellos al Fondo Común Municipal. Mayor autonomía en la generación de recursos promueve la búsqueda de mejores estrategias de gestión de sus recursos, pudiendo alcanzar una mayor cobertura en la entrega de los servicios públicos que satisfagan de forma más óptima las necesidades y requerimientos de sus ciudadanos.

Asimismo, ante las restricciones que enfrentan las políticas de presupuesto a nivel comunal en Chile, que reducen las atribuciones de la autoridad del Gobierno local en materia presupuestaria, (Acuña et al., 2019), el uso eficiente de estos recursos cobra especial importancia. De esta manera, y debido a restricciones institucionales, la política de gasto municipal chilena es probable que sea independiente de las preferencias de los votantes o del alcalde en ejercicio. Lo anterior hace indispensable garantizar una gestión eficiente de los recursos municipales, con el objeto de liberar recursos y contribuir a la expansión del gasto social. Los resultados sugieren que el éxito en estas estrategias de gestión estaría condicionado a la configuración política del concejo municipal y la orientación política del Gobierno central de turno. Para poder desligar el desempeño del Gobierno local en términos de eficiencia en el uso de los recursos desde la influencia política, es necesario promover instancias formales de articulación entre los servicios públicos y los municipios, que promuevan espacios de colaboración y sinergias entre la gestión local y central, que no discrimine por coalición política. 


\section{Bibliografía}

Acuña, H., Carrasco, D., Carrasco, M., \& Soto, A. (2019). "El efecto del partido político del alcalde sobre variables de gasto municipal". El Trimestre Económico, 86(342), 343-404.

Afonso, A., \& Fernandes, S. (2006). "Measuring local government spending efficiency: Evidence for the Lisbon region". Regional Studies, 40(1), 39-53.

Aigner, D., Lovell, C. A. K., \& Schmidt, P. (1977). "Formulation and estimation of stochastic frontier production function models". Journal of Econometrics, 6(1), 21-37.

Balaguer-coll, M. T., Prior, D., \& Tortosa-ausina, E. (2007). "On the determinants of local government performance: A two-stage nonparametric approach". European Economic Review, 51(2), 425-451.

Balaguer-coll, M. T., Prior, D., \& Tortosa-ausina, E. (2013). "Output complexity, environmental conditions, and the efficiency of municipalities". Journal of Productivity Analysis, 39(3), 303-324.

Banker, R. D., Charnes, A., \& Cooper, W. W. (1984). "Some Models For Estimating Thechnical And Scale Inefficiencies In Data Envelopment Analysis". Managment Science, 30(9), 1078-1092.

Benito, B., Bastida, F., \& García, J. A. (2010). "Explaining differences in efficiency: an application to Spanish municipalities". Applied Economics, 42(4), 515-528.

Borger, B. De, \& Kerstens, K. (1996). "Cost efficiency of Belgian local governments: A comparative analysis of FDH , DEA , and econometric approaches". Regional Science and Urban Economics, 26, 145-170.

Bosch, N., \& Espasa, M. (2012). "Citizen control and the efficiency of local public services". Environment and Planning C, 30, 248-266.

Bostan, I., Onofrei, M., Popescu, C., Lupu, D., \& Firtescu, B. (2018). "Efficiency and Corruption in Local Counties: Evidence from Romania". Lex Localis, 16(2), 215-229.

Cavalcante, P. (2013). "La competencia electoral genera un gobierno más eficiente? Un estudio comparativo de los municipios de Brasil". Revista de Administración Pública, 47(6), 1569-1591.

Charnes, A., Cooper, W. W., \& Rhodes, E. (1978). "Measuring the efficiency of decision making units". European Journal of Operational Research, 2(6), 429-444.

Chumacero, R. \& Paredes, R. (2012). "Centralización, focalización y consecuencias no deseadas: Evaluación de los programas de empleo en Chile". Economía Chilena, 15(1), 4-18.

Cordero, J. M., Pedraja-Chaparro, F., Pisaflores, E. C., \& Polo, C. (2017). "Efficiency assessment of Portuguese municipalities using a conditional nonparametric approach". Journal of Productivity Analysis, 48(1), 1-24.

Da Cruz, N. F., \& Marquez, R. C. (2014). "Revisiting the determinants of local government performance". Omega, 44, 91-103. 
Díaz Castro, J., \& de Jorge Moreno, J. (2018). "Análisis de la eficiencia y factores explicativos de la gestión de los municipios del Meta, Colombia”. Revista Finanzas y Política Económica, 10(1), 211-234.

El Mehdi, R. \& Hafner, C.M. (2014). "Local Government Efficiency: The Case of Moroccan Municipalities". African Development Review, 26(1), 88-101.

Färe, R., \& Primont, D. (1995). Multi-Output Production and Duality: Theory and Applications. Boston: Kluwer Academic Publishers.

Farrell, M. J. (1957). "The Measurement of Productive Efficiency". Journal of the Royal Statistical Society, 120(3), 253-290.

Fernández Santos, Y., \& Flórez López, R. (2006). “Aplicación del modelo dea en la gestión pública. Un análisis de la eficiencia de las capitales de provincia españolas". Revista Iberoamericana de Contabilidad de Gestión, (7), 165-202.

Greene, W. (2005a). "Reconsidering heterogeneity in panel data estimators of the stochastic frontier model". Journal of Econometrics, 126: 269-303.

Greene, W (2005b). "Fixed and random effects in stochastic frontier models". Journal of Productivity Analysis, 23: 7-32.

Hayashi, F. Econometrics. (2000). Princeton: Princeton University Press.

Henríquez Díaz, M., \& Fuenzalida Aguirre, J. (2011). “Compensando la desigualdad de ingresos locales: El Fondo Común Municipal (FCM) en Chile". Revista Iberoamericana de Estudios Municipales, (4), 73-104.

Hollingsworth, B, Dawson, P. J, Maniadakis, N. (1999). "Efficiency measurement of health care: a review of non-parametric methods and applications". Health Care Management Science, 2 (3), 161-172.

Kalseth, J., \& Ratts $\varnothing$, J. (1995). "Spending and overspending in local government administration: A minimum requirement approach applied to Norway”. European Journal of Political Economy, 11, 239-251.

Lampe, H. W., \& Hilgers, D. (2015). "Trajectories of efficiency measurement: A bibliometric analysis of DEA and SFA". European Journal of Operational Research, 240(1), 1-21.

Ley No 18.695 Diario Oficial de la República De Chile, Santiago, Chile, 26 de julio de 2006.

Ley No 19.816 Diario Oficial de la República De Chile, Santiago, Chile, 07 de agosto de 2002.

Ley No 20.033 Diario Oficial de la República De Chile, Santiago, Chile, 01 de julio de 2005.

Ley No 20.237 Diario Oficial de la República de Chile, Santiago, Chile, 24 de diciembre de 2007.

Ley No 20.410 Diario Oficial de la República de Chile, Santiago, Chile, 20 de enero de 2010.

Loikkanen, H. A., \& Susiluoto, I. (2005). “Cost Efficiency of Finnish Municipalities in Basic Service Provision 1994-2002”. Urban Public Economics Review, 4, 39-64.

Morales-Trapp, S. (2016). "Participación ciudadana y accountability: reflexiones sobre la experiencia comparada en Chile y Colombia". Revista Jurídicas, 13(2), 100-113. 
Nijkamp, P., \& Suzuki, S. (2009). “A Generalized Goals-achievement Model in Data Envelopment Analysis: an Application to Efficiency Improvement in Local Government Finance in Japan”. Spatial Economic Analysis, 4(3), 249-274.

O'Donnell, C. J. (2011). The Sources of Productivity Change in the Manufacturing Sectors of the U.S. Economy (07/2011). Brisbane.

Pacheco, F., Sanchez, R., \& Villena, M. (2014). A Longitudinal Parametric Approach to Estimate Local Government Efficiency. Documento de trabajo, Dirección de Presupuesto del Ministerio de Hacienda, Santiago de Chile.

Papke, L. E., \& Wooldridge, J. M. (1996). "Econometric Methods For Fractional Response Variables With An Application To 401 (K) Plan Participation Rates". Journal Of Applied Econometrics, 11(February), 619-632.

Papke, L. E., \& Wooldridge, J. M. (2008). "Panel data methods for fractional response variables with an application to test pass rates". Journal of Econometrics, 145, 121-133.

Pérez, M. (2016). “Análisis de los municipios chilenos: ingresos por gestión versus transferencias del Fondo Común Municipal". Estudios de Políticas Públicas, 4, 121-130.

Raupp, F. M., \& de Pinho, J. A. G. (2013). “Accountability em câmaras municipais: uma investigação em portais eletrônicos". Revista de administração, 48(4), 770-782.

Renne, R. R. (1937). "Farm Taxation: Research in Measuring the Efficiency of Local Governments". American Journal of Agricultural Economics, 19(2), 553-557.

Sampaio de Sousa, M. C., Cribari-Neto, F., \& Stosic, B. D. (2005). "Explaining DEA Technical Efficiency Scores in an Outlier Corrected Environment: The Case of Public Services in Brazilian Municipalities". Brazilian Review of Econometrics, 25(2), 287-313.

Sickles, R. C., \& Zelenyuk, V. (2019). Measurement of Productivity and Efficiency. Cambridge University Press.

Silva, C., \& Crisóstomo, V. (2019). "Gestão fiscal, eficiência da gestão pública e desenvolvimento socioeconômico dos municípios cearenses". Revista de Administração Pública, 53(4), 791-801.

Silva, C., Alves, R., De Luca, M., \& Vasconcelos, A. (2019). "Eficiência da alocação de recursos públicos nas unidades da federação nos governos Lula e Dilma". Cadernos Gestão Pública e Cidadania, 24(78), 1-20.

Soto, I. (2016). "El Efecto de la Competencia Política sobre la Provisión de Bienes Públicos Locales en México”. Revista de Ciencia Política, 36(3), 749-772.

Št’astná, L., \& Gregor, M. (2015). "Public sector efficiency in transition and beyond: evidence from Czech local governments". Applied Economics, 47(7), 680-699.

Chamberlin, G. (1980). "Analysis of variance with qualitative data". The Review of Economic Studies, 47, (1), 225-238. 
Wooldridge, J. M. (2010). Econometric Analysis of Cross Section and Panel Data. (P. MIT, Ed.) (second edi).

Worthington, A. C. (2000). “Cost Efficiency In Australian Local Government : A Comparative Analysis Of Mathematical Programming And Econometric Approaches". Financial Accountability \& Management, 16(3), 201-223.

Yusfany, A. (2015). "The Efficiency Of Local Governments And Its Influence Factors". International Journal of Scientific \& Technology Research, 4(10), 219-241. 


\section{Anexos}

TABLA A1

EFECTOS MARGINALES MODELO PROBIT FRACCIONAL

\begin{tabular}{|c|c|}
\hline \multirow{2}{*}{ VARIABLES } & (6) \\
\hline & ET \\
\hline Población & $\begin{array}{c}0.089 * * * \\
(0.0217)\end{array}$ \\
\hline Part_IPP & $\begin{array}{c}0.083^{* * *} \\
(0.018)\end{array}$ \\
\hline Part_FCM & $\begin{array}{c}0.191 * * * \\
(0.023)\end{array}$ \\
\hline Población_Rural & $\begin{array}{c}-0.090 * * * \\
(0.020)\end{array}$ \\
\hline Func_Profesional & $\begin{array}{c}-0.016^{* *} \\
(0.006)\end{array}$ \\
\hline Cambio_Alcalde & $\begin{array}{l}-0.006 \\
(0.005)\end{array}$ \\
\hline Alcalde_Candidato & $\begin{array}{l}0.007 * \\
(0.003)\end{array}$ \\
\hline Alcalde_Concert & $\begin{array}{l}-0.007 \\
(0.019)\end{array}$ \\
\hline Consejales_Concert & $\begin{array}{l}-0.019 \\
(0.029)\end{array}$ \\
\hline Gobierno_Concert & $\begin{array}{c}-0.066^{* * *} * \\
(0.014)\end{array}$ \\
\hline Consejales_Concert*Alcalde_Concert & $\begin{array}{c}0.014 \\
(0.019)\end{array}$ \\
\hline Alcalde_Concert*Gobierno_Concert & $\begin{array}{c}0.002 \\
(0.008)\end{array}$ \\
\hline Consejales_Concert*Gobierno_Concert & $\begin{array}{c}0.025^{* *} \\
(0.011)\end{array}$ \\
\hline Alcalde_Concert*Consejales_Concert*Gobierno_Concert & $\begin{array}{l}-0.004 \\
(0.008)\end{array}$ \\
\hline Dummy por año & $\mathrm{Si}$ \\
\hline Log seudo verosimilitud & -270.3628 \\
\hline Heterogeneidad individual & $\mathrm{Si}$ \\
\hline Observaciones & 1,631 \\
\hline
\end{tabular}

Fuente: elaboración propia a partir de los resultados. ${ }^{*} * * \mathrm{p}<0.01, * * \mathrm{p}<0.05, * \mathrm{p}<0.1$ 
TABLA A2

RESULTADOS MODELO PROBIT FRACCIONAL CONSIDERANDO RETORNOS VARIABLES DE ESCALA

\begin{tabular}{|c|c|c|c|c|c|c|}
\hline \multirow{2}{*}{ VARIABLES } & (1) & (2) & (3) & (4) & (5) & (6) \\
\hline & ET & ET & ET & ET & ET & ET \\
\hline \multirow[t]{2}{*}{ Población } & $0.00124 * * *$ & $0.00121 * * *$ & $0.00122 * * *$ & $0.00119^{* * *}$ & $0.00119 * * *$ & $0.00267 * * *$ \\
\hline & $(0.000374)$ & $(0.000370)$ & $(0.000372)$ & $(0.000371)$ & $(0.000371)$ & $(0.000983)$ \\
\hline \multirow[t]{2}{*}{ Part_IPP } & -0.000529 & -0.00107 & -0.00109 & -0.00116 & -0.00117 & -0.000307 \\
\hline & $(0.00190)$ & $(0.00194)$ & $(0.00191)$ & $(0.00190)$ & $(0.00190)$ & $(0.00202)$ \\
\hline \multirow[t]{2}{*}{ Part_FCM } & -0.000457 & -0.000982 & -0.000856 & -0.000846 & -0.000845 & $0.00495 * * *$ \\
\hline & $(0.00144)$ & $(0.00147)$ & $(0.00145)$ & $(0.00144)$ & $(0.00144)$ & $(0.00142)$ \\
\hline \multirow[t]{2}{*}{ Población_Rural } & 0.00121 & 0.00115 & 0.00111 & 0.00106 & 0.00106 & 0.000892 \\
\hline & $(0.000892)$ & $(0.000893)$ & $(0.000887)$ & $(0.000891)$ & $(0.000890)$ & $(0.000895)$ \\
\hline \multirow[t]{2}{*}{ Func_Profesional } & -0.000595 & -0.000409 & -0.000285 & -0.000275 & -0.000317 & 0.000134 \\
\hline & $(0.00100)$ & $(0.000951)$ & $(0.000946)$ & $(0.000948)$ & $(0.000953)$ & $(0.000819)$ \\
\hline Cambio_Alcalde & & & $-0.0705^{*}$ & -0.0679 & -0.0684 & -0.0612 \\
\hline \multirow[t]{2}{*}{ Alcalde_Candidato } & & & $0.0803 * *$ & $\begin{array}{l}(0.0429) \\
0.0775^{*}\end{array}$ & $\begin{array}{l}(0.0428) \\
0.0784 *\end{array}$ & $0.0846 * *$ \\
\hline & & & $(0.0399)$ & $(0.0403)$ & $(0.0403)$ & $(0.0398)$ \\
\hline \multirow[t]{2}{*}{ Alcalde_Concert } & & & 0.00159 & -0.0483 & -0.108 & -0.101 \\
\hline & & & $(0.0349)$ & $(0.115)$ & $(0.118)$ & $(0.119)$ \\
\hline \multirow[t]{2}{*}{ Consejales_Concert } & & & -0.000357 & -0.00221 & -0.00267 & $-0.00313^{*}$ \\
\hline & & & $(0.00154)$ & $(0.00184)$ & $(0.00186)$ & $(0.00179)$ \\
\hline \multirow[t]{2}{*}{ Gobierno_Concert } & & & $-0.0881^{* *}$ & $-0.139 * *$ & $-0.171^{* *}$ & $-0.208^{* *}$ \\
\hline & & & $(0.0405)$ & $(0.0681)$ & $(0.0810)$ & $(0.0816)$ \\
\hline Consejales_ & & & & 0.00271 & 0.00444 & 0.00428 \\
\hline Concert*Alcalde_Concert & & & & $(0.00303)$ & $(0.00311)$ & $(0.00312)$ \\
\hline \multirow{2}{*}{$\begin{array}{l}\text { Alcalde_Concert*Gobierno_ } \\
\text { Concert }\end{array}$} & & & & $-0.104 * *$ & 0.0283 & 0.0146 \\
\hline & & & & $(0.0442)$ & $(0.127)$ & $(0.127)$ \\
\hline & & & & 0.00269 & 0.00382 & $0.00422 *$ \\
\hline Concert*Gobierno_Concert & & & & $(0.00188)$ & $(0.00241)$ & $(0.00241)$ \\
\hline \multirow{4}{*}{$\begin{array}{l}\text { Alcalde_} \\
\text { Concert*Consejales__ } \\
\text { Concert*Gobierno_Concert }\end{array}$} & & & & & -0.00394 & -0.00355 \\
\hline & & & & & & \\
\hline & & & & & & \\
\hline & & & & & $(0.00377)$ & $(0.00375)$ \\
\hline \multirow[t]{2}{*}{ Constant } & $1.733 * * *$ & $1.856^{* * *}$ & $1.860^{* * * *}$ & $1.910^{* * *}$ & $1.925^{* * *}$ & $2.272^{* * *}$ \\
\hline & $(0.113)$ & $(0.116)$ & $(0.124)$ & $(0.126)$ & $(0.127)$ & $(0.223)$ \\
\hline Dummy por Año & No & $\mathrm{Si}$ & $\mathrm{Si}$ & $\mathrm{Si}$ & $\mathrm{Si}$ & $\mathrm{Si}$ \\
\hline Log seudo verosimilitud & -199.8827 & -198.5995 & -198.4410 & -198.3152 & -198.2999 & -198.0257 \\
\hline Heterogeneidad individual & No & No & No & No & No & $\mathrm{Si}$ \\
\hline Observaciones & 1,631 & 1,631 & 1,631 & 1,631 & 1,631 & 1,631 \\
\hline
\end{tabular}

Los errores estándar robustos están entre paréntesis $* * * \mathrm{p}<0.01, * * \mathrm{p}<0.05, * \mathrm{p}<0.1$

Fuente: elaboración propia a partir de los resultados. 
TABLA A3

RESULTADOS MODELO FRONTERA ESTOCÁSTICA DE COSTOS

\begin{tabular}{|c|c|c|}
\hline VARIABLES & Coeficiente & $\begin{array}{l}\text { Errores estándar } \\
\text { robustos }\end{array}$ \\
\hline \multicolumn{3}{|l|}{ Frontera estocástica } \\
\hline Asist_Esc & $0.174 * * *$ & 0.0610 \\
\hline Cons_APS & $0.0844 * * *$ & 0.0186 \\
\hline Area_Verd & $0.0264 * * *$ & 0.00883 \\
\hline Sup_Pobr & $0.179 *$ & 0.0940 \\
\hline \multicolumn{3}{|l|}{ Variables explicativas } \\
\hline Población & -0.000922 & 0.000890 \\
\hline Part_IPP & $-0.00648 * * *$ & 0.00175 \\
\hline Part_FCM & $-0.00772 * * *$ & 0.00156 \\
\hline Población_Rural & $0.0141 * *$ & 0.00659 \\
\hline Func_Profesional & $0.000839 *$ & 0.000499 \\
\hline Cambio_Alcalde & -0.00682 & 0.0218 \\
\hline Alcalde_Candidato & 0.0280 & 0.0203 \\
\hline Gobierno_Candidato & 0.938 & 0.931 \\
\hline Consejales_Concert & 0.000606 & 0.000914 \\
\hline Alcalde_Concert & 0.108 & 0.0801 \\
\hline Consejales_Concert*Alcalde_Concert & -0.00281 & 0.00194 \\
\hline Alcalde_Concert*Gobierno_Concert & -0.0103 & 0.125 \\
\hline Consejales_Concert*Gobierno_ & $-0.00221^{*}$ & 0.00119 \\
\hline $\begin{array}{l}\text { Concert } \\
\text { Alcalde Concert*Consejales }\end{array}$ & 0.00134 & 0.00323 \\
\hline \multicolumn{3}{|l|}{ Concert*Gobierno_Concert } \\
\hline Constante & $-4.352 * * *$ & 0.276 \\
\hline$\delta^{2}$ & 0.0810346 & \\
\hline$\delta^{2}$ & 0.1134963 & \\
\hline$\lambda$ & 0.7139849 & \\
\hline Dummy por año & $\mathrm{Si}$ & \\
\hline Log seudo verosimilitud & 898.7987 & \\
\hline Observaciones & 233 & \\
\hline
\end{tabular}

Nota $* * * \mathrm{p}<0.01, * * \mathrm{p}<0.05, * \mathrm{p}<0.1$

Fuente: elaboración propia a partir de los resultados. 
TABLA A4

RESULTADOS MODELO PROBIT FRACCIONAL SIN INCORPORAR LA VARIABLE POBREZA EN LA FRONTERA

\begin{tabular}{|c|c|c|c|c|c|c|}
\hline \multirow{2}{*}{ VARIABLES } & (1) & (2) & (3) & (4) & (5) & (6) \\
\hline & ET & ET & ET & ET & ET & ET \\
\hline \multirow[t]{2}{*}{ Población } & $0.00223^{* * *}$ & $0.00227 * * *$ & $0.00226^{* * *}$ & $0.00225^{* * *}$ & $0.00225^{* * *}$ & $0.00377 * * *$ \\
\hline & $(0.000622)$ & $(0.000631)$ & $(0.000645)$ & $(0.000643)$ & $(0.000643)$ & $(0.00103)$ \\
\hline \multirow[t]{2}{*}{ Part_IPP } & 0.00420 & $0.00493^{*}$ & $0.00579 * *$ & $0.00571^{* *}$ & $0.00570 * *$ & $0.00776 * * *$ \\
\hline & $(0.00278)$ & $(0.00283)$ & $(0.00274)$ & $(0.00273)$ & $(0.00273)$ & $(0.00189)$ \\
\hline \multirow{2}{*}{ Part_FCM } & $0.0122^{* * *}$ & $0.0130 * * *$ & $0.0134 * * *$ & $0.0134 * * *$ & $0.0134 * * *$ & $0.0111^{* * *}$ \\
\hline & $(0.00213)$ & $(0.00218)$ & $(0.00207)$ & $(0.00207)$ & $(0.00207)$ & $(0.00139)$ \\
\hline \multirow[t]{2}{*}{ Población_Rural } & $-0.00652 * * *$ & $-0.00648^{* * *}$ & $-0.00634 * * *$ & -0.00636 *** & $-0.00637 * * *$ & $-0.00633^{* * *}$ \\
\hline & $(0.00148)$ & $(0.00149)$ & $(0.00143)$ & $(0.00144)$ & $(0.00144)$ & $(0.00148)$ \\
\hline \multirow[t]{2}{*}{ Func_Profesional } & -0.000697 & -0.000873 & -0.000585 & -0.000594 & -0.000622 & $-0.00142 * *$ \\
\hline & $(0.00122)$ & $(0.00121)$ & $(0.00119)$ & $(0.00119)$ & $(0.00121)$ & $(0.000656)$ \\
\hline Cambio_Alcalde & & & $\begin{array}{l}-0.0673 \\
(0.0564)\end{array}$ & $\begin{array}{l}-0.0636 \\
(0.0569)\end{array}$ & $\begin{array}{l}-0.0638 \\
(0.0569)\end{array}$ & $\begin{array}{l}-0.0679 \\
(0.0559)\end{array}$ \\
\hline \multirow[t]{2}{*}{ Alcalde_Candidato } & & & $0.0928^{*}$ & $0.0884^{*}$ & $0.0889^{*}$ & $0.0922 *$ \\
\hline & & & $(0.0499)$ & $(0.0502)$ & $(0.0502)$ & $(0.0496)$ \\
\hline \multirow[t]{2}{*}{ Alcalde_Concert } & & & 0.0319 & -0.0227 & -0.0627 & -0.0840 \\
\hline & & & $(0.0486)$ & $(0.165)$ & $(0.173)$ & $(0.174)$ \\
\hline \multirow[t]{2}{*}{ Consejales_Concert } & & & $0.00462^{* *}$ & 0.00213 & 0.00182 & 0.00171 \\
\hline & & & $(0.00201)$ & $(0.00248)$ & $(0.00254)$ & $(0.00250)$ \\
\hline \multirow[t]{2}{*}{ Gobierno_Concert } & & & $-0.243^{* * *}$ & $-0.368 * * *$ & $-0.388^{* * *}$ & $-0.399 * * *$ \\
\hline & & & $(0.0454)$ & $(0.0832)$ & $(0.0883)$ & $(0.0884)$ \\
\hline Consejales_ & & & & 0.00201 & 0.00315 & 0.00371 \\
\hline Concert*Alcalde_Concert & & & & $(0.00450)$ & $(0.00476)$ & $(0.00478)$ \\
\hline \multirow{2}{*}{$\begin{array}{l}\text { Aldent } \\
\text { Concert }\end{array}$} & & & & -0.0340 & 0.0522 & 0.0822 \\
\hline & & & & $(0.0570)$ & $(0.175)$ & $(0.172)$ \\
\hline \multirow{2}{*}{$\begin{array}{l}\text { Consejales_____Concert } \\
\text { Concert*Gobierno_C }\end{array}$} & & & & $0.00438 * *$ & $0.00511^{* *}$ & $0.00532^{* *}$ \\
\hline & & & & $(0.00207)$ & $(0.00241)$ & $(0.00239)$ \\
\hline \multirow{4}{*}{$\begin{array}{l}\text { Alcalde_} \\
\text { Concert*Consejales__ } \\
\text { Concert*Gobierno_Concert }\end{array}$} & & & & & -0.00254 & -0.00320 \\
\hline & & & & & & \\
\hline & & & & & & \\
\hline & & & & & $(0.00511)$ & $(0.00501)$ \\
\hline \multirow[t]{2}{*}{ Constante } & $-0.509 * * *$ & $-0.540 * * *$ & $-0.750^{* * * *}$ & $-0.673^{* * *}$ & $-0.662 * * *$ & $-0.872 * *$ \\
\hline & $(0.177)$ & $(0.178)$ & $(0.176)$ & $(0.181)$ & $(0.185)$ & $(0.385)$ \\
\hline Dummy por año & No & $\mathrm{Si}$ & $\mathrm{Si}$ & $\mathrm{Si}$ & $\mathrm{Si}$ & $\mathrm{Si}$ \\
\hline Log seudo verosimilitud & -760.4953 & -756.6259 & -754.1245 & -753.7474 & -753.7255 & -753.3096 \\
\hline Heterogeneidad individual & No & No & No & No & No & $\mathrm{Si}$ \\
\hline Observaciones & 1,631 & 1,631 & 1,631 & 1,631 & 1,631 & 1,631 \\
\hline
\end{tabular}

Los errores estándar robustos están entre paréntesis $* * * \mathrm{p}<0.01, * * \mathrm{p}<0.05, * \mathrm{p}<0.1$

Fuente: elaboración propia a partir de los resultados. 


\section{FIGURA A1}

LÍNEA DE TIEMPO ELECCIONES PRESIDENCIALES Y MUNICIPALES EN CHILE PERIODO 2010-2016

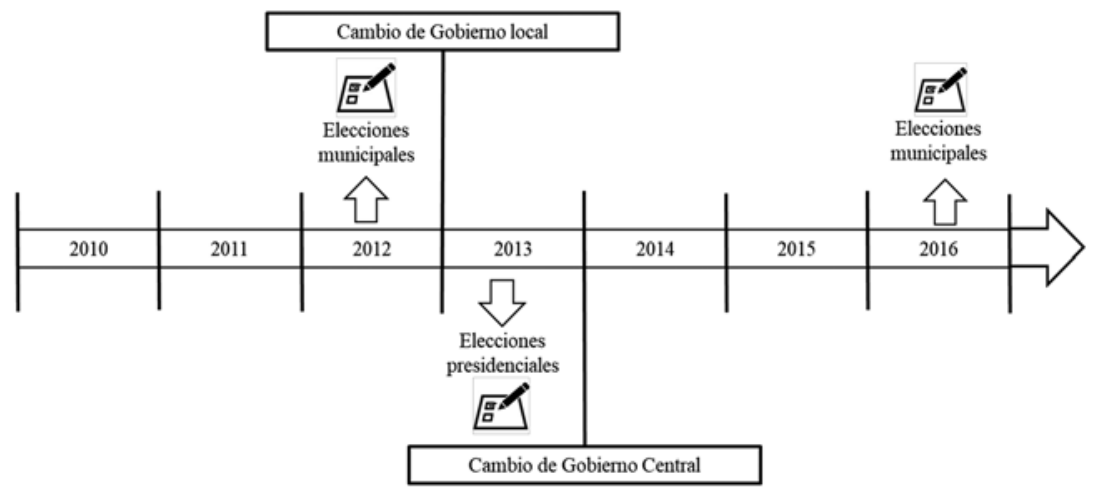

Fuente: elaboración propia. 